\title{
Gamification of Assembly Planning in Virtual Environment
}

\section{Purpose:}

The objective of the research is to study the effect of the gamification of virtual assembly planning on user performance, user experience, and engagement.

\section{Design/methodology/approach}

A multi-Touch table was used to manipulate virtual parts and gamification features were integrated into the virtual assembly environment. An experiment was conducted in two conditions: a gamified and a nongamified virtual environment. Subjects had to assemble a virtual pump. The user performance was evaluated in terms of the number of errors, the feasibility of the generated assembly sequence and the user feedback.

\section{Findings:}

The gamification reduced the number of errors and increased the score representing the number of right decisions. The results of the subjective and objective analysis showed that the number of errors decreased with engagement in the gamified assembly. The increase in the overall user experience reduced the number of errors. The subjective evaluation showed a significant difference between the gamified and the nongamified assembly in terms of the level of engagement, the learning usability, and the overall experience.

\section{Limitations}

The effective learning retention after training has not been tested and longitudinal studies are necessary. The effect of the used gamification elements has been evaluated as a whole, further work could isolate the most beneficial features and add other elements that might be more beneficial for learning.

\section{Originality:}

The research reported in this paper provides valuable insights into the gamification of virtual assembly using a low-cost multi-touch interface. The results are promising for training operators to assemble a product at the design stage. 


\section{Introduction}

The assembly activity is vital in the manufacturing industry, as it represents almost $40 \%$ of the cost of the product (Hongbo et al., 2006). The product assembly plan is the sequence of assembly operations that connect parts together to make a functional product. Before the emergence of virtual reality technologies, the generation of the product assembly plan was obtained by performing assembly trials using physical prototypes of parts. The assembly operators had to take note of the assembly sequences they obtain when connecting real parts until they find the most feasible (optimum) assembly plan. The ease of assembly/disassembly and the time taken to assemble the product are the main criteria of comparison of the assembly sequences.

Virtual Assembly (VA) is based on the use of the computer-generated environment to assemble 3D models of parts. Testing the product "assemblability" at the design stage is important to check the feasibility of assembly operations and for the generation of its assembly plan. Additionally, assembling virtual parts is important for the designer to refine the product design based on the information obtained from the assembly trials and the degree of the feasibility of the assembly sequences generated from the interaction with VE (Boothroyd, Geoffrey. Dewhurst, Peter. Knight, 1994; Santochi and Dini, 1992). Hence, the manipulation of the digital prototypes of parts allows the optimization of the product design to ease its assembly/disassembly (A. Delchambre, 1992; Zorriassatine et al., 2003). Moreover, proactive production planning, resource allocation, assembly time and cost estimation, assembly operators' training and maintenance planning and costing can be made at the early stage of the product lifecycle. Hence, virtual assembly allows optimizing the product development cycle and significantly shortens time-to-market, considered as an important key issue in nowadays-worldwide industrial competition (Garbaya et al., 2003). To assemble an object both the visual form and the instructions are necessary to accomplish the task correctly (Hodgins, 2006). From a given number of possible assembly operations, the determination of the optimum assembly sequence allows reducing the assembly time and cost; game mechanics addressed these aspects with Goal-Oriented Action Planning (GOAP) (Yu et al., 2012).

Digital games are originally created for entertainment, and the American game industry made a scintillating sale of \$25 billion dollars in 2010 (Muntean, 2011). However, this has drastically changed with the amalgamation of games with teaching in recent years opening a plethora of opportunities. The positive impact on learning and mood supports games adoption in many domains (M.Nazry and Romano, 2017). The use of game elements in non-gaming context, also termed as gamification, enhances users' experience and engagement and has caught the interest of marketers (Deterding et al., 2011), game developers (Cheong et al., 2013), researchers (McGonigal, 2011), military personnel (Fardo, 2013) and many other domain experts from varied fields (Groh, 2012). The gamification concept has been used for effective learning and training purposes for a while, for example in Serious Games (Neill, 2009); or to enhance knowledge acquisition in virtual worlds (Bellotti et al., 2008). Due to the growing interest in academic research, detailed research was conducted by (Hamari et al., 2014). Seen in popular applications, like FourSquare, the design methodology to gamify an artifact consists of adding game-like elements (Anderson et al., 2013). Possible game-like elements to be used for gamification are badges, leaders boards, points, time pressure; having a progress bar; adopting a theme; stimulating exploration; discovery; curiosity and emotions; showing the consequences of, for example, team building \& collaborative and/or competitive task; or social activities, are more fun and contribute to gamify an artifact. Hence, many popular object and service have incorporated gamification into their core design process (e.g. iPhone and apps). 
Considering the role of gamification and its impact on learning, the present research aims to verify whether the use of gamification of a virtual assembly task provides an advantage to the users in terms of the number of errors made in connecting parts and the generation of feasible assembly sequences. Also, the user experience and engagement in the assembly task are investigated. A virtual assembly environment was created and a comparative study was conducted between the assembly task execution with and without gamification. The paper is organized as follows: Section 2 provides the related literature in virtual assembly planning and the gamification concept. The aim and the hypothesis of this research are summarised in section 3. Following, the methodology utilized to validate the virtual assembly system is presented in section 4 . The results are presented in section 5. The discussion, the limitations of this research and the conclusion are presented in sections 6,7 and 8 , respectively.

\section{Related literature}

\subsection{Virtual Assembly Planning}

The research in virtual assembly planning appeared since the emergence of virtual reality. The benefit of manipulating virtual porotypes of parts to generate the assembly sequence of products was proved for the optimization of the product design and for the ease of assembly. (Christiand and Yoon, 2011) developed a system called "Intelligent Virtual Assembly System" in which a simple genetic algorithm provides optimal paths for haptic guidance and the assembly sequence of the parts to be assembled. The paper reports on the application of this approach to the planar assembly by combining both optimizations of path planning and assembly sequence. The evaluation of the system showed a significant reduction of the assembly time and the travel distance of the assembly task executed with haptic guidance to follow the optimal path and the assembly sequence generated by the algorithm.

(Xia et al., 2013) published a survey of the research on virtual reality and haptics for product assembly ranging from rigid bodies to soft cables. The authors consider that aspects of virtual assembly such as modeling and visualization, collision detection, assembly sequence and path planning are almost mature, however, the issues such as haptics interaction, constraint simulation, physics modeling, data integration, and tolerance quality are not very well developed and only a few applications are adopted in industry. The analysis of the key technologies for virtual assembly revealed that future research directions should focus on the design of tools and fixtures, DFA evaluation, ergonomics evaluation, knowledge, and intelligence as these issues are important but still not mature and not included in the current industrial applications.

(Abidi et al., 2015) developed a haptic virtual reality platform aiming to plan, perform and evaluate virtual assemblies. They used the phantom desktop device as haptic and 3D interface and a single screen for the visualization. The dynamic behavior of the virtual parts was obtained with the PhysX engine and they used OpenGL graphics library for the visualization of the assembly scene. A virtual handle represented by a cone in the scene was used to manipulate parts. When there is a contact between the manipulated object and another object in the VE, a force is calculated and rendered to the user through the haptic device. The authors carried out a case study of blower house assembly, they concluded that haptic feedback is important for enhancing the sense of presence and it is beneficial for virtual assembly task. However, the paper did not present a study of performance metrics to evaluate their approach and the process of fitting parts together is not detailed in the paper.

(Marzano et al., 2015) developed a virtual reality framework called VR_MATE for maintainability and assembly analysis of complex systems. VR_MATE integrates VR hardware and software; a simulation manager was included to customize the system's architecture that covers a wide range of interface devices. The system allows the operator (performing both assembly and maintenance) to execute actions by combining the product knowledge base with the criteria required for specific operations. The system was evaluated by two case studies: the maintainability tests and the assembly analysis of an aircraft carriage and a railway coach cooling system. The results showed that VR_MATE generated the disassembly sequence and showed that parts are detachable and accessible. The authors did not provide details about the interaction between parts and subassemblies during the assembly/disassembly operations. 
(Wang et al., 2016) considered that the assumptions made by previous research in computing the force rendered to the user through haptic devices did not provide the user with a realistic feeling of forces during the execution of assembly operations. They developed an algorithm that computes the assembly force for three basic mechanical fit types, namely clearance fit, interference fit, and transition fit. The author considered that their algorithm allowed rendering to the operator fast, stable, and more realistic assembly force at real-time during the Virtual Assembly (VA) operation.

(Zhou et al., 2016) proposed a method that uses swept volumes (SV) to graphically represent ergonomic information for maintainability design process in virtual environments. The authors considered that traditional evaluation methods of verification of the adequacy of maintenance space, based on physical prototypes and expert knowledge, are time-consuming and influenced by subjectivity. They developed a framework made of five modules: support data, virtual environment, verification method, output, and feasible applications. In order to evaluate maintenance operations, they developed a verification technique based on the swept volume generated by the operator's manual activity and the human factors/ergonomics knowledge (HFE). The system was evaluated by a maintenance case study of a Boeing 737 auxiliary power unit. The results of the space maintenance evaluation allowed to identify the limitation of the reserved space to operate a screw on the power unit. The authors considered their proposed method is feasible and efficient as shown by the case study they carried out.

(Li et al., 2016) defined hand gestures for grasping parts in the virtual assembly. They developed a gesture recognition algorithm for grasping four types of mechanical parts. A constraint navigation approach was adopted to guide the user in assembling parts. The authors implemented a glove-based virtual assembly system named (GBVAS) using the CyberGlove II, an immersive display made of a pair of polarized stereoscopic glasses and a FASTRAK as a tracking system. When executing the task, the user had to follow the assembly sequence provided by the assembly planning module and he is guided by displaying text information to start current assembly operation and by blinking the color of the part to be assembled. The system was evaluated by conducting a case study that involved 80 subjects to assemble a two-stage gear reducer made of more than 40 parts. The authors reported that the feedback collected from the users showed that the proposed gesture recognition system provided natural interaction and the efficiency of the virtual assembly was improved. However, the authors did not provide the details about the evaluation factor representing the assembly efficiency.

(Carlson Patrick et al., 2016) carried out a user study, which involved 52 subjects, to explore the effectiveness of various interaction device configurations for bimanual virtual assembly. They created 5 configurations using two devices: the Phantom Omni ${ }^{\circledR}$ haptic device and the 5DT Data Glove. The task consisted of the insertion of one virtual part into another for which the user performance was measured by the task completion time. For qualitative evaluation, the participants were asked to provide their appreciation about each configuration they used. Although the results of this study did not reveal a significant difference in performance between the five interaction conditions, the use of the Glove in the non-dominant hand and the Omni in the dominant hand for bimanual assembly resulted in similar performance when compared to the other configurations tested for this specific task. The subjective evaluation showed that $50 \%$ of the participants chose the 5DT Dara Glove and the haptic-enabled device as their preferred interaction configuration.

(Gallegos-Nieto et al., 2017) investigated the transfer of knowledge and skills from haptic-enabled virtual assembly environment to real-world assembly. The training platform was created upon the basis of HAMS (Haptic Assembly and Manufacturing System) developed by (Gonzalez-Badillo et al., 2014). The authors conducted a study involving three groups of subjects and three training modes: (VA) with haptic feedback, (VA) without haptic and training by watching a video. Each group was trained to perform several assembly tasks with increasing complexity and number of parts; after the training session, the subjects were requested to perform the task in the real world. The results indicated that, compared to training by video, the group of subjects that were trained with (VA) had up to $80 \%$ improvement in the performance of the real assembly task, expressed by the Task Completion Time (TCT). Moreover, haptic-enabled (VA) training led to greater levels of effectiveness than without haptics and training with (VA) is more effective for more complex assembly task. 
(Sagardia and Hulin, 2017) presented a virtual assembly platform that supports bimanual haptic feedback for the manipulation of complex objects. Their system used a bimanual haptic device that displays six-DoF force feedback; it is made of two DLR/KUKA light-weight robot arms and an additional force-torque sensor at each robot wrist. They developed a collision detection engine based on a combination of penalty-and constraint-based force rendering methods. The authors claimed that their study aims at solving the virtual assembly challenging problems such as realistic and practical scenarios including complex geometries, bimanual six-DoF haptic interaction for hands and arms, and intuitive navigation in large workspaces. The results of testing the system for the assembly of a car control box showed that it is able to handle complex scenarios with multiple geometries imported directly from CAD environments. The system allowed large upper body movements and provided force and tactile feedback to the hand and the forearm.

(Wang et al., 2017) proposed a new model for simulating forces for virtual assembly tasks of mechanical parts with a clearance fit. The assembly operation was decomposed into three consecutive states: free navigation state, positioning state and assembly state. When a collision between the manipulated part and the receiving part occurs, the collision force is simulated and the assembly process enters the positioning state. For enhancing the user perception, the authors developed a model of the force rendered during the assembly state which they applied to the case of assembling a shaft and its bushing. To evaluate the usability of the system, they conducted an experiment involving 15 participants with four experimental conditions. The subjects were asked to assemble four sets of shaft-bushing with clearance fits and the user performance was measured. The results showed that shorter task completion time (TCT) was obtained without force rendering but the realism was very low in this condition. However, the authors claimed that the force rendering model allowed better immersion and realism of the virtual assembly task.

Most of the previous research integrated complex and high-cost interfaces tending to mimic the shop floor interactions. However, despite their proven usefulness for the application for which they were developed, the accessibility of these systems is very limited due to the cost barrier and the lack of their versatility. The approach reported in this paper is an alternative solution based on simple and easy to use interfaces for assembly planning. The system includes a multi-touch table, the physical engine NVIDIA PhysX for collision detection and for modeling the dynamic behavior of parts. Gamification elements were included in the VA to enhance the user experience and motivation to generate valid assembly plans.

\subsection{Gamification concept}

While the term 'Gamification' may hold the same aesthetical meaning at its core, some authors define it as a use of game design elements in non-game context (Deterding et al., 2011). Other authors address it as a process of game-thinking and mechanics to help engage users and solve problems (Malone, 1980). It is also termed as a process for enhancing services driven by the motivation to invoke gameful experiences and future behavioral outcomes (Hamari et al., 2014). Having been used in a number of applications, the concept of adding game mechanics helps users to learn, solve problems and inculcate new ideas and concepts in their everyday lives. Gamification was implemented in the business sector to introduce concepts that would otherwise be boring and tedious to learn. For example, Cisco uses game elements to teach the concept of binary numbers and IBM uses a thinking game to help understand its employees the idea of process management. Over the past recent years, a new wave of interest was made by industries and marketing professionals. They have tactfully implemented the same through social networking medium (Facebook, Twitter, etc.), games (Temple Run, Candy Crush, etc.), location-based services (Foursquare), image and video sharing services (Instagram, Snapchat, etc.) and many others. In the early 1980s, self-learning paradigm through enjoyable interfaces of games had already been studied (Bellotti et al., 2008). Recently, the standardized design patterns that promote heuristics came into play. The aim of using gamification is not to impart an enjoyable experience only, but also to serve a purpose. The game theory has grown in the past few years, from identifying design principles that promote motivation in a collaborative environment (Zhang, 2008), to recognizing pure physiological motivation in video games (Ryan et al., 2006). 
A growing body of research looks into "games with a purpose" looping back as gameplay to solve human information tasks. This included work detailing specific design features that afford player enjoyment (Cheng, 1995; Sy et al., 2011). Furthermore, researchers in HCI and management sciences have identified design principles that enhance the motivational affordances of computer-supported collaborative work principles (Groh, 2012; Yu, 2003), which are congruent with research on the motivational psychology of video games (Pellens et al., 2005). In persuasive technology (Fardo, 2013), video games and game aspects have been studied as a technology to define the user behavior according to the intention of the system designer (Neill, 2009; Southey and Linders, 2001), or to infuse embedded values (Deterding et al., 2011). Socio-psychological studies on online interactions of communities and the motivational aspects of recommender systems showed the importance of the core design properties of video games (Anderson et al., 2013; Bille et al., 2004). It is suggested that the reward and reputation systems of gamified applications should be modeled with economically inspired approaches such as Incentive-Centred Design. The user experience of video games is considered a major issue of HCI, with developing new methodologies, models and intelligent interactivity for the usability or playability of games (Arvo and Novins, 2000; Cheong et al., 2013; Olsen et al., 2009). However, there is little research about the benefits of gamification in learning and education. For example, Nor \& Romano (2017) have found that learning with a gamified virtual environment significantly improves learners' mood (M.Nazry and Romano, 2017). The meaning of gamification, how it can be used and the possible misuse are explained in (Anderson et al., 2013). The authors defined gamification as the use of game mechanics, dynamics and frameworks to promote desired behaviors. (Hou and Ramani, 2006) explained how gamification could be used to identify behaviors and correct them, thus investigating the benefits of gamification in learning and e-learning. (Cheong et al., 2013) noticed a significant increase in the gamification of learning and instruction. The integration of game elements such as time, accuracy and point systems into gamified training programs encourage users to achieve their desired goals.

A more extended approach to address work distribution among multidisciplinary teams using game mechanics was studied (Lewis and Mistree, 1997). User Experience (UX) and user engagement through game mechanics have opened up new ways of performing activities. This could be beneficial to the industry in the domain of assembly planning. An attempt to automate assembly planning of mechanical parts by using artificial intelligence (AI) is presented in (Yu et al., 2012), By using Goal-Oriented Action Planning (GOAP) principle, the system generates a topological planning graph based on partial information to obtain an optimum assembly plan. The principle consists of four key entities: the goal, which defines the ultimate achievable condition; the plan, which consists of a sequence of feasible steps; the action, which defines a single feasible step within a plan and the formulation, which defines a methodical strategy to achieve a goal. At a given point, there may be a number of different formulations to achieve a goal, but only the formulation with the lowest cost function is selected. (Lim et al., 2007) presented the factors that affect the user performance of an assembly system that includes haptic feedback. They concluded that factors such as the size of parts and stereovision could effective assembly using the gamification concept.

\section{Aim and hypothesis}

The use of gamification in executing an assembly task could potentially lead to better engagement and enjoyment in the task with an improved understanding and learning. However, it is unclear whether users'

performance and engagement in a virtual assembly task (considering the number of time the sequences have been followed correctly and the number of errors) will also be affected by the introduction of gamification. A comparative experimental study was carried out with the following research question:

RQ1 Does the introduction of gamification improves task performance in multitouch-based virtual assembly?

RQ2: Does the introduction of gamification, in a virtual assembly task, improves user engagement, experience, and perceived learning? 
We hypothesize that this will be the case and that gamification will improve both task performance, user's experience and engagement.

\section{Methodology}

\subsection{Virtual assembly environment}

The virtual assembly environment was generated by a system called Collaborative Conceptual Design Environment (Co-DIVE) where the design of virtual objects is based on sketching and matching. The programmer and the domain expert participate collaboratively in the creation of the virtual environment. The Co-DIVE system is made of three main elements: the sketching block, the VR builder and the Attribute Creation and Modification (ACM) module. It includes a simple graphical user interface (GUI) implemented in multi-touch a table, it allows manipulating objects, adding semantics and adding interactive attributes. Figure 1 presents the working structure of the Co-DIVE application.

Figure 1. The architecture of the Co-DIVE application

The sketching block serves as the starting point of the Co-DIVE system (Figure 2.a). The designer begins by sketching his/her idea of the object, then, the matching process is launched to generate the 3D models of parts from a database, Figure 2.b. The visualization of the virtual objects is made by the VR builder block, Figure 2.c. The process of sketching can be repeated a number of times, generating desired models, to populate the VR builder which will then use these objects to describe the entire virtual assembly scene. The interactive properties of the parts were defined by using high-level semantics. Once the scene is completed, the application generates an equivalent .X3D format of the scene, preserving the included semantics, the kinematics and physical properties of objects (Figure 2.c).

Figure 2. (a) Sketching block, (b) Selection of matched objects, (c) VR builder block.

\subsection{Participants}

Sixteen participants from various backgrounds and gender (14 males, 2 females), age ranging from 22 to 44 years old (Mean $\pm S D 29.12 \pm 6.55$ ) were recruited via social media and word of mouth, they included academics, students, and the general population.

All subjects were right-handed, $20 \%$ of them wore corrective glasses, $45 \%$ stated they were using a virtual environment for the very first time, 70\% that they had never performed an assembly task before, and 55\% that they were aware of the concept of serious gaming. 


\subsection{Material}

A multi-touch table was utilized for the experiment to interact with the objects displayed on the screen through the use of touch, providing a more intuitive method of manipulation of the virtual objects. The assembly application was running on Windows 8 operating system (Figure 3).

\subsection{Tools}

\subsubsection{Pre-Questionnaire}

A pre-exposure questionnaire enquired about the following items: gender, whether the participant is left or right-handed, previous experience with virtual environments, previous experience with games, serious games and/or assembly systems.

\subsubsection{Post-Questionnaire}

A questionnaire composed of eleven items enquiring on the self-reported engagement and performances during the task was created. In particular, it enquired about the following concept asking the user to score on 10 points Likert-like scale, where 0 was low/or very poor, 5 was neutral and 10 was high/ very good:

- How engaged were you while performing the assembly / playing the game?

- Was the overall assembly task difficult to achieve?

- Was the use of the multi-touch table helpful while performing the assembly?

- Did you feel you learned something about assembling mechanical parts from this?

- How was the overall experience of using the system?

For the gamified versions of the experiment, three further items where added:

- Did the game elements like score, errors made and remaining time have an effect on your performance?

- Was your performance led by the additional incentives like bonus points?

- Did you use the hints during the game for achieving the assembly task?

The questionnaire was used in an equivalent French version and statistical tests were performed to analyze the collected data.

Figure 3. A user engaged in the assembly game on the multi-touch table

\subsubsection{Gamification process}

The use of game mechanics in a learning environment was detailed in (Igarashi et al., 1999; Shilane et al., 2004), and similar principles have been developed and integrated into the virtual assembly described in this paper. The following gamification elements were included:

- Points,

- Hints

- Incentive messages

- Time pressure

- An environment stimulating exploration and discovery

- The consequences of the user's action in the virtual assembly system were highlighted by the assignation of a point or an error. 
The gamification block was integrated the Co-DIVE system, as shown in Figure 4. Once the user generated a 3D model of the virtual assembly scene, the gamification module is deployed. In the game, a user has to generate feasible assembly sequences and assemble a pump system with the aid of the gamification features.

Figure 4. The integration of the gamification concept in Co-DIVE system

The task consists of assembling five different parts to obtain a functional product. The users had to interact with one of two types of applications: The virtual assembly environment coupled with game mechanics or the virtual assembly environment without gaming. In the gamification condition, the player receives points when parts are assembled correctly (that is s/he has followed a feasible assembly sequence), while an error is counted when a wrong assembly operation is made. An action of the user is considered an error when he/she joins parts without respecting the precedence relationships, which could hinder the assembly of other parts of the pump. In addition, the player receives for bonus scores, badges, and hints throughout the gameplay.

Figure 5. Gamification of the virtual assembly environment

The gamified assembly begins by presenting the users with a welcome message; it contains menus displaying the information about the mechanical components (Figure 5). 3D models of the parts are displayed in the virtual scene. Before launching the game, the user has to enter his/her profile such as the name and age. A timer is set to 5 minutes to get the pump assembled. The user begins by grabbing and joining parts together. Some basic rules for the game were defined (Table 1). The scoring system depends on the adherence of the user to these rules and his/her strategy of sequencing the actions of joining parts. Physically based modeling and collision detection among parts were implemented to support the notification of errors. In addition, timely hints during the games help users to take corrective actions in joining parts; they also assist the users when they tend to become stuck at any point during assembly activity.

Table 1. Game rules

\begin{tabular}{|l|l|}
\hline No. & \multicolumn{1}{c|}{ Rule } \\
\hline 1 & Click the Start button to begin the countdown timer \\
\hline 2 & You get a total of 5 minutes to complete the game \\
\hline 3 & For each correct assembly, you score 10 points \\
\hline 4 & There are no negative points \\
\hline 5 & Get a bonus of 30 points for completing the whole assembly \\
\hline 6 & At the end of the timer, you stop the game \\
\hline 7 & Get a "Star Assembler" title when you score a total of 50 points \\
\hline 8 & Use hints in between game to assist in assembly \\
\hline
\end{tabular}


Figure 6.a represents the gamified version of the virtual assembly environment and Figure 6.b represents the non-gamified version. In the non-gamified condition of the experiment, the game mechanics were stripped to allow virtual assembly scene but the performance data such as the obtained score and the assembly errors were automatically saved for evaluation purposes.

Figure 6. (a) Non-Gamified virtual assembly; (b) Gamified virtual assembly

\subsection{Data Collection}

Relevant data such as Total score, Total number of errors, were collected for each of the two groups, although the score, time and error were not visible to the user in the non-gamified condition. In addition, for the group utilizing the gamified version, the number of hints used was also recorded. Also, the rating provided by the users on the post-experience questionnaire were recorded.

\subsection{Procedure}

After explicating the informed consent procedure and filling the pre-test, a training session of 20 minutes followed, during which the users were shown the actual pump assembly plan, which they could relate to the components on the multi-touch table.

The users were informed about the different gestures, assigned by the multi-touch API that could help them to manipulate and join the mechanical parts. They were also made aware of the scoring system, the error counter, the gaming timer and the available hints in the gamified condition.

During the training session, no records were made of the scores, errors nor hints provided to the volunteers. Following the training, the volunteers were asked to assemble a pump made of 5 parts and assigned at random to one of the two conditions: a) pump assembly with the gamified virtual environment or b) pump assembly in the virtual environment without gamification. At the end of the assembly task, the subjects were asked to fill the post-exposure questionnaire.

The experimental procedure is summarized in table 2 below. 
Table 2. Experiment condition of virtual assembly environment

\begin{tabular}{|c|c|}
\hline Task description & $\begin{array}{l}\text { Manipulate the 3D parts and assemble the pump model in order to generate feasible } \\
\text { assembly sequences }\end{array}$ \\
\hline Model & Unrelated Design \\
\hline Subjects & $\begin{array}{l}\text { Sixteen volunteers: } \\
-\quad 8 \text { assigned to Gamified assembly environment } \\
-\quad 8 \text { assigned to the non-gamified assembly environment }\end{array}$ \\
\hline $\begin{array}{l}\text { Experimental } \\
\text { conditions }\end{array}$ & $\begin{array}{l}\text { Two experimental conditions: } \\
\text { 1. Gamified virtual assembly environment running on Windows } 8 \\
\text { Mode of interaction: } \\
\text { - } \quad \text { multi-touch table } \\
\text { - } \quad \text { Session time set to } 5 \text { minutes } \\
\text { - } \quad \text { Includes: notification of errors, bonus for correct actions (scores and } \\
\text { - } \quad \text { badges), hints for taking corrective actions when the user is stuck } \\
\text { OR } \\
\text { 2. } \quad \text { Virtual assembly environment running on the same platform } \\
\text { Mode of interaction: } \\
\text { - } \quad \text { multi-touch table } \\
\text { - Session time set to } 5 \text { minutes } \\
\text { - The scores and the errors are automatically saved by the system }\end{array}$ \\
\hline $\begin{array}{l}\text { Experimental } \\
\text { factors }\end{array}$ & Gamified and non-gamified assembly environment \\
\hline $\begin{array}{l}\text { Experimental } \\
\text { measures }\end{array}$ & $\begin{array}{l}\text { Objective evaluation for both experimental conditions } \\
\text { - } \quad \text { Scores and the number of errors in executing the assembly task } \\
\text { For the gamified assembly only: } \\
-\quad \text { Number of hints requested } \\
\text { Subjective analysis based on the feedback from users: } \\
\text { - For both experimental conditions: } \\
\text { - } \quad \text { Perception of the task difficulty } \\
\text { - } \quad \text { Estimation of the user engagement } \\
\text { - } \quad \text { The usefulness of the multi-touch table for the assembly task } \\
\text { - } \quad \text { Pexception } \\
\text { - } \quad \text { Perception of the overall experience } \\
\text { For the gamified assembly only: } \\
\text { - } \quad \text { The effect of the gamification elements } \\
\text { - } \text { The usefulness of the hints } \\
\text { - } \quad \text { The user engagement in the gameplay } \\
\text { - } \quad \text { Obtaining the bonus points at the end of the game }\end{array}$ \\
\hline
\end{tabular}

\section{Results}

The analysis of the data collected from the experiment carried out in the gamification-based assembly and the non-gamified version of the virtual assembly yielded the results described in the following sections. 


\subsection{Objective Analysis}

The errors and scores obtained in the two experimental conditions were automatically recorded in the background of the application (Table 3).

\subsubsection{Number of errors made during assembly}

The Shapiro Wilk test was carried out on the collected data to check the normality of the data distribution. The probability values obtained $($ Game $(\mathrm{U}:=8)=0.37$, Without Game $(\mathrm{U}:=8)=$ 0.58 ) clearly indicates that the collected are normally distributed.

Table 3. Error count for the two experimental conditions

\begin{tabular}{|l|l|llllllll}
\hline Condition & Mean \& Std. Dev. & \multicolumn{1}{|l|}{ Number of Errors } \\
\hline With Game & $2.625 \pm 1.060$ & 2 & 1 & 2 & 4 & 3 & 4 & 3 & 2 \\
\hline Without Game & $5.500 \pm 3.116$ & 8 & 2 & 11 & 4 & 6 & 2 & 4 & 7 \\
\hline
\end{tabular}

An independent sample T-test test was carried out on the number of errors. The results indicated that there is a significant difference in the number of errors made under the gamification condition $(\mathrm{M}=2.625, \mathrm{SD}=1.060)$ and without gamification condition $(\mathrm{M}=5.500, \mathrm{SD}=3.116) ; \mathrm{t}(14)=-$ $2.470, \mathrm{p}=0.027$. The average number of errors made during the non-gamified assembly was significantly higher than in the gamified assembly. The graphic plot presented in Figure 7 shows that the gamified assembly reduced the number of errors in generating the assembly sequence.

Figure 7. Mean level for errors in the two experimental conditions

\subsubsection{Score obtained in the assembly task}

The Shapiro Wilk test was carried out on the collected data set presented in Table 4 to confirm the normality of data distribution. The probability values obtained (Game $(\mathrm{U}:=8)=0.11$, Without Game $(\mathrm{U}:=8)=0.36)$ indicated that the data sets are normally distributed.

Table 4. Score count for the two experimental conditions

\begin{tabular}{|l|l|llllllll|}
\hline Condition & Mean \& Std. Dev. & \multicolumn{8}{|l|}{ Score (8 users/condition) } \\
\hline With Game & $35.00 \pm 22.677$ & 20 & 30 & 70 & 30 & 30 & 10 & 20 & 70 \\
\hline Without Game & $22.50 \pm 20.528$ & 20 & 10 & 10 & 30 & 10 & 10 & 20 & 70 \\
\hline
\end{tabular}

A T-test was performed, it indicates a significant difference between the two conditions: $\mathrm{t}(14)=$ $1.156, p=0.267$. The diagram of Figure 8 shows that the gamified assembly condition significantly increased the score obtained by the participants. This indicates that subjects performed the pump assembly with correct sequences more readily with gamification than without gamification. 
Figure 8. Mean level for scores in the two experimental conditions

\subsubsection{Number of hints utilized}

The users in the gamified versions utilized on average $3.25( \pm 1.83)$ hints of a maximum of ten hints available. A simple linear regression analysis was conducted to predict the score obtained during the gamified assembly based on the number of hints utilized. A significant regression equation was found $(\mathrm{F}(1,6)=1.001, \mathrm{p}<0.356)$, with $\mathrm{R}^{2}=.000$; indicating that the number of hints utilized is a predictor of the score that will be obtained. The hints' predicted score is equal to $19.787+0.4681$ (errors) hints when the number of errors is measured as the number of hints utilized, participants' error decreased of 0.4681 for each hint utilized. Additionally, a simple linear regression was conducted between the number of errors during the gamified assembly and the number of hints utilized. The number of hints utilized is only marginally a predictor of the errors made, where an only marginally significant regression equation was found $(\mathrm{F}(1,6)=4.509$, $\mathrm{p}<$ .078 ). The hints' predicted error is equal to $1.479+.468$ (errors) hints when errors are measured as the number of hints utilized. Participants' error decreased by 0.468 for each hint utilized.

\subsection{Subjective-Objective Analysis}

A Pearson correlation function was used to evaluate conditions of linear dependence based on the errors the users made and their self-reported level of engagement and the overall experience.

- Engagement for the gamified assembly: The diagram of Figure 9 indicates that there is a negative correlation between the variables of error and engagement $(r(8)=-0.71, p=0.04)$. This suggests that error has the tendency to decrease with increasing engagement in the gamified assembly.

Figure 9. The negative correlation between error and engagement in the gamified condition

- Overall Experience in the gamified assembly: The diagram shows that there is a slight negative correlation between error and overall experience $(r(8)=-0.18, p=0.67)$. This suggests that when the overall experience increases the number of errors decreases in the gamified assembly Figure 10. 
Figure 10. The negative correlation between error and overall experience in the gamified condition

Additionally, the participants were asked to give an evaluation of their engagement, assembly difficulty, learning usability, multi-touch table usefulness and the rating to the overall experience. Figure 11 presents the scores collected from the subjects under both experimental conditions. Each of the items enquired was scored in a Likert-like scale from 0 (low) to 10 (high) and 5 is the middle point. The results of the statistical analysis of the collected data are discussed below. As these were stepped scores the parametric Mann-Witney test has been utilized for all the questions.

Figure 11. Feedback from users

- Q1 - Engagement: the Mann-Whitney U-test shows that there is a significant difference in the level of engagement between the conditions with and without gamification [U-U (16); $\mathrm{h}=1, \mathrm{p}=0.00015$, ranksum $=100]$.

- Q2 - Assembly Difficulty: there is no significant difference between the gamified and nongamified condition as assessed with a Mann-Whitney U-Test [U-U (16); $h=0, p=0.64$, ranksum $=63]$.

- Q3 - Multi-touch Usefulness: there is no significant difference between the gamified and non-gamified condition as assessed with a Mann-Witney U-Test [U-U (16); $h=0, p=0.33$, ranksum=78].

- Q4 - Perceived Learning: the Mann-Whitney U-test shows that there is a significant difference between the gamified and non-gamified condition based on the criteria of learning usability [U-U (16); $\mathrm{h}=1, \mathrm{p}=0.00015$, ranksum=100].

- Q5 - Overall Experience: the Mann-Whitney U-test shows that there is a significant difference in the overall experience with and without gamification [U-U (16); $h=1$, $\mathrm{p}=0.00015$, ranksum $=100]$.

Figure 12. Users' scoring of the assembly game 
In addition, the subjects who used the gamified virtual assembly were asked to rate how they perceived the effect of the game elements in the game, and this was rated positive (having obtained a score of 6.75 over the middle point 5 ). They also found the additional incentive provided by the gamification useful (score of 6.37, higher than middle point 5). Additionally, the perceived number of hints utilized was 5.87, which is higher than the actual number of hints utilized (as recorded by the game) of $3.25( \pm 1.83)$ hints of a maximum of ten hints available (Figure 12).

\section{Discussion}

In line with (Hanus and Fox, 2015), it was found that the gamification of the virtual assembly task improves the users' engagement with the task, the perception of having learned something, and the overall experience. The assembly difficulty is perceived to be the same with and without gamification, which is a good result as the assembly task was the same and the introduction of gamification did not complicate it. In addition, the use of a multi-touch table was perceived the same under both conditions, again proving that the introduction of gamification did not change the modality under which the task was executed.

The user made significantly less error and achieved a higher score in the gamified assembly, demonstrating that a gamified virtual assembly is a better system for learning. Interestingly learners on average overestimated the number of hints utilized, and despite the average perception of the players of having used more than half of the hints available, the effective number of hints utilized on average was very low and in most case less than half of the number of hints available. However, the gamification led to a significantly better performance in term of errors made, as well as the correct number of actions that the participants made correctly. In addition, the results showed a clear positive correlation between the number of errors made and the score of engagement in the task as well as the overall experience, showing that while gamification helps in achieving better performances, in turn, this helps engagement and the overall experience is perceived even more positively.

These results are beneficial for the designers and creators of virtual assembly training environments. This confirms that despite the utilization of a multi-touch table for the manipulation of virtual objects, gamification makes a difference in performance and self-perceived engagement and learnability of the training. As such, it is suggested that gamification should be utilized in a system that aims to provide training on how to assemble an artifact.

\section{Limitations}

The study has provided some significant results despite the relatively modest number of users that have been utilized in each condition. The effective learning retention after the training has not been tested and longitudinal studies could be conducted. Also, only some gamification elements have been introduced in the virtual assembly environment and their effect has been evaluated as a whole. Further work could try to isolate which of the feature chosen is most beneficial and is there any other gamification elements (e.g. social elements) which could also be even more beneficial for the learning, as for example suggested by (Sailer et al., 2017). Furthermore, a multi-touch table was utilized for the evaluation, but the benefits of the gamification of assembly planning activity could also be tested utilizing low-cost interactive devices such as tablets and mobile phones. Future research and development could also evaluate the assembly of more complex mechanical systems that contain a very large number of components. 


\section{Conclusion}

A virtual assembly environment using the multi-touch interactive device was developed. The objective is to generate the product assembly sequence at the design stage. In an attempt to enhance the user experience and engagement, and the learnability of the assembly task, the virtual assembly environment was coupled with game mechanics. The gamified virtual assembly was evaluated against a virtual environment without gamification. Based on the results obtained from the objective analysis it can be concluded that the use of gamification is to be suggested in virtual assembly training environments. The gamified assembly significantly allowed reducing the number of errors when joining parts together. It increased the score representing the number of correct actions made during assembly planning activity. The results of the subjective and objective analysis showed that the number of errors decreased with engagement in the gamified assembly. In addition, the increase in the overall user experience reduces the number of errors. Moreover, the subjective evaluation showed a significant difference between the gamified and non-gamified assembly environment in terms of the level of engagement, the learning usability, and the overall experience.

\section{REFERENCES}

A. Delchambre. (1992), Computer-Aided Assembly Planning, edited by Chapman \& Hall, 1992nd ed., Springer, Dordrecht, available at:https://doi.org/https://doi.org/10.1007/978-94-011-2322-8.

Abidi, M.H., Ahmad, A., Darmoul, S. and Al-Ahmari, A.M. (2015), "Haptics assisted virtual assembly", IFAC-PapersOnLine, Vol. 28, pp. 100-105.

Anderson, A., Huttenlocher, D., Kleinberg, J. and Leskovec, J. (2013), "Steering user behavior with badges", Proceedings of the 22nd International Conference on World Wide Web - WWW'13, pp. 95-106.

Arvo, J. and Novins, K. (2000), "Fluid sketches: continuous recognition and morphing of simple handdrawn shapes", Proceedings of the 13th Annual ACM Symposium on ..., Vol. 2, pp. 73-80.

Bellotti, F., Berta, R., De Gloria, A. and Zappi, V. (2008), "Exploring gaming mechanisms to enhance knowledge acquisition in virtual worlds", Proceedings of the 3rd International Conference on Digital Interactive Media in Entertainment and Arts - DIMEA '08, p. 77.

Bille, W., Pellens, B., Kleinermann, F. and De Troyer, O. (2004), "Intelligent modelling of virtual worlds using domain ontologies", Proceedings of the IVEVA 2004 Workshop, No. February 2013, pp. 272279.

Boothroyd, Geoffrey. Dewhurst, Peter. Knight, W.A. (1994), Product Design for Manufacture and Assembly, Marcel Dekker, Inc.

Carlson Patrick, and Vance, J.M. and and Berg Meisha. (2016), "An evaluation of asymmetric interfaces for bimanual virtual assembly with haptics", Virtual Reality, Vol. 20 No. 4, pp. 193-201.

Cheng, Y. (1995), "Mean Shift, Mode Seeking, and Clustering", IEEE Transactions on Pattern Analysis and Machine Intelligence, Vol. 17 No. 8, pp. 790-799.

Cheong, C., Cheong, F. and Filippou, J. (2013), "Quick Quiz: A Gamified Approach for Enhancing Learning", PACIS 2013 Proceedings, pp. 1-14. 
Christiand and Yoon, J. (2011), "Assembly simulations in virtual environments with optimized haptic path and sequence", Robotics and Computer-Integrated Manufacturing, available at:https://doi.org/10.1016/j.rcim.2010.07.015.

Deterding, S., Dixon, D., Khaled, R. and Nacke, L. (2011), "From game design elements to gamefulness: Defining gamification", Proceedings of the 15th International Academic MindTrek Conference on Envisioning Future Media Environments - MindTrek'11, pp. 9-11.

Fardo, M.L. (2013), "The gamification of learning and instruction: game-based methods and strategies for training and education", Conjectura: Filos. Educ, Vol. 18 No. 1, pp. 201-206.

Gallegos-Nieto, E., Medellín-Castillo, H.I., González-Badillo, G., Lim, T. and Ritchie, J. (2017), “The analysis and evaluation of the influence of haptic-enabled virtual assembly training on real assembly performance", International Journal of Advanced Manufacturing Technology, Vol. 89 No. 1-4, pp. 581-598.

Garbaya, S., Coiffet, P. and Blazevic, P. (2003), "Experiments of assembly planning in virtual environment", Proceedings of the IEEE International Symposium on Assembly and Task Planning, Vol. 2003-Janua, pp. 85-89.

Gonzalez-Badillo, G., Medellin-Castillo, H., Lim, T., Ritchie, J. and Garbaya, S. (2014), "The development of a physics and constraint-based haptic virtual assembly system", Assembly Automation, Vol. 34 No. 1, pp. 41-55.

Groh, F. (2012), "Gamification: State of the Art Definition and Utilization", Research Trends in Media Informatics, pp. 39-46.

Hamari, J., Koivisto, J. and Sarsa, H. (2014), "Does gamification work? - A literature review of empirical studies on gamification", Proceedings of the Annual Hawaii International Conference on System Sciences, pp. 3025-3034.

Hanus, M.D. and Fox, J. (2015), "Assessing the effects of gamification in the classroom: A longitudinal study on intrinsic motivation, social comparison, satisfaction, effort, and academic performance", Computers and Education, Vol. 80, pp. 152-161.

Hodgins, H.W. (2006), "The Future of Learning Objects", Educational Technology, Educational Technology Publications, Inc., Vol. 46 No. 1, pp. 49-54.

Hongbo, S., Shuxia, L.I., Degang, G. and Peng, L.O.U. (2006), "Genetic simulated annealing algorithmbased assembly sequence planning", International Technology and Innovation Conference 2006 (ITIC 2006), Vol. 2006, pp. 1573-1579.

Hou, S. and Ramani, K. (2006), "Sketch-based 3D Engineering Part Class Browsing and Retrieval", Proceedings of the EUROGRAPHICS Workshop on Sketch-Based Interfaces and Modeling, pp. 131-138.

Igarashi, T., Matsuoka, S. and Tanaka, H. (1999), “Teddy: a sketching interface for 3D freeform design”, Proceedings of the 26th Annual Conference on Computer Graphics and Interactive Techniques SIGGRAPH '99, pp. 409-416.

Lewis, K. and Mistree, F. (1997), "Modeling interactions in multidisciplinary design: A game theoretic approach", AIAA Journal, Vol. 35 No. 8, pp. 1387-1392.

Li, J., Xu, Y., Ni, J. and Wang, Q. (2016), "Glove-based virtual hand grasping for virtual mechanical assembly”, Assembly Automation, Emerald, Vol. 36 No. 4, pp. 349-361.

Lim, T., Ritchie, J.M., Dewar, R.G., Corney, J.R., Wilkinson, P., Calis, M., Desmulliez, M., et al. (2007), "Factors affecting user performance in haptic assembly", Virtual Reality, Vol. 11 No. 4, pp. 241252.

M.Nazry, N.N. and Romano, D.M. (2017), "Mood and learning in navigation-based serious games", Computers in Human Behavior, Vol. 73 No. 73, pp. 596-604.

Malone, T.W. (1980), "What makes things fun to learn? heuristics for designing instructional computer games", Proceedings of the 3rd ACM SIGSMALL Symposium and the First SIGPC Symposium on Small Systems - SIGSMALL '80, pp. 162-169.

Marzano, A., Friel, I., Erkoyuncu, J.A. and Court, S. (2015), "Design of a virtual reality framework for maintainability and assemblability test of complex systems", Procedia CIRP, 37, pp. 242-247. 
McGonigal, J. (2011), "Reality is Broken: Why Games Make Us Better and How They Can Change the World", New York, p. 402.

Muntean, C.C.I. (2011), "Raising engagement in e-learning through gamification", The 6th International Conference on Virtual Learning ICVL 2011, No. 1, pp. 323-329.

Neill, T. (2009), "Serious games: Learning for the generation", edited by Gimson, A.Development and Learning in Organisations, Emerald Group Publishing Limited, Vol. 23 No. 4, pp. 12-15.

Olsen, L., Samavati, F.F., Sousa, M.C. and Jorge, J.A. (2009), "Sketch-based modeling: A survey", Computers and Graphics (Pergamon), Pergamon, Vol. 33 No. 1, pp. 85-103.

Pellens, B., Bille, W., De Troyer, O. and Kleinermann, F. (2005), "VR-wise: A conceptual modelling approach for virtual environments", Methods and Tools for Virtual Reality (MeTo-VR 2005) Workshop, Vol. 2 No. 1, pp. 1-10.

Ryan, R.M., Rigby, C.S. and Przybylski, A. (2006), "The motivational pull of video games: A selfdetermination theory approach", Motivation and Emotion, Vol. 30 No. 4, pp. 347-363.

Sagardia, M. and Hulin, T. (2017), "Multimodal Evaluation of the Differences between Real and Virtual Assemblies", IEEE Transactions on Haptics, pp. 1-12.

Sailer, M., Hense, J.U., Mayr, S.K. and Mandl, H. (2017), "How gamification motivates: An experimental study of the effects of specific game design elements on psychological need satisfaction", Computers in Human Behavior, available at:https://doi.org/10.1016/j.chb.2016.12.033.

Santochi, M. and Dini, G. (1992), Computer-Aided Planning of Assembly Operations. The Selection of Assembly Sequences, Vol. 9.

Shilane, P., Min, P., Kazhdan, M. and Funkhouser, T. (2004), “The Princeton Shape Benchmark”, Proceedings - Shape Modeling International SMI 2004, pp. 167-178.

Southey, F. and Linders, J.G. (2001), "Ossa-A Conceptual Modelling System for Virtual Realities", Conceptual Structures: Broadening the Base: 9th International Conference on Conceptual Structures, \{ICCS $\}$ 2001, Stanford, \{CA\}, \{USA\}, July 30-August 3, 2001, Proceedings, Springer, Berlin, Heidelberg, Vol. 9, p. 333.

Sy, S., Zichermann, G. and Cunningham, C. (2011), Gamification by Design: Implementing Game Mechanics in Web and Mobile Apps, Strategic Synergy, available at:https://doi.org/10.1093/intimm/dxs132.

Wang, Q.H., Huang, Z.D., Li, J.R. and Liu, J.W. (2017), “A force rendering model for virtual assembly of mechanical parts with clearance fits", Assembly Automation, available at:https://doi.org/10.1108/AA-12-2016-175.

Wang, Q.H., Huang, Z.D., Ni, J.L., Xiong, W. and Li, J.R. (2016), “A novel force rendering approach for virtual assembly of mechanical parts", International Journal of Advanced Manufacturing Technology, Vol. 86 No. 1-4, pp. 977-988.

Xia, P., Lopes, A.M. and Restivo, M.T. (2013), "A review of virtual reality and haptics for product assembly: From rigid parts to soft cables", Assembly Automation, available at:https://doi.org/10.1108/01445151311306672.

Yu, B. (2003), "Recognition of Freehand Sketches Using Mean Shift", IUI '03 Proceedings of the 8th International Conference on Intelligent User Interfaces, ACM, Miami, Florida, USA, pp. 204-210.

Yu, H., Lim, T., Ritchie, J., Sung, R., Louchart, S., Stnescu, I.A., Roceanu, I., et al. (2012), "Exploring the application of computer game theory to automated assembly", Procedia Computer Science, Vol. 15, pp. 266-273.

Zhang, P. (2008), "Technical opinion: Motivational affordances: reasons for ICT design and use", Communications of the ACM, Vol. 51 No. 11, pp. 145-147.

Zhou, D., Chen, J., Lv, C. and Cao, Q. (2016), "A method for integrating ergonomics analysis into maintainability design in a virtual environment", International Journal of Industrial Ergonomics, Vol. 54, pp. 154-163.

Zorriassatine, F., Wykes, C., Parkin, R. and Gindy, N. (2003), "A survey of virtual prototyping techniques for mechanical product development", Proceedings of the Institution of Mechanical Engineers, Part B: Journal of Engineering Manufacture, IMECHE, Vol. 217 No. 4, pp. 513-530. 


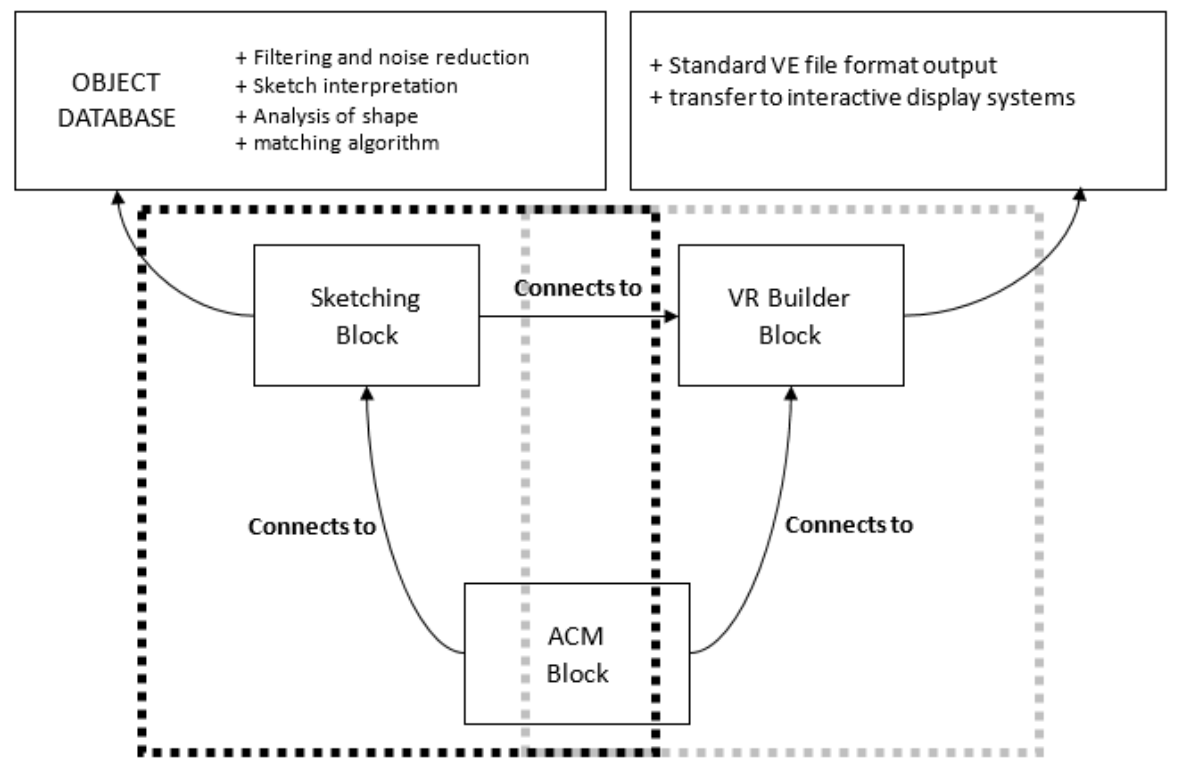

Figure 1: The architecture of the Co-DIVE application 
(a)

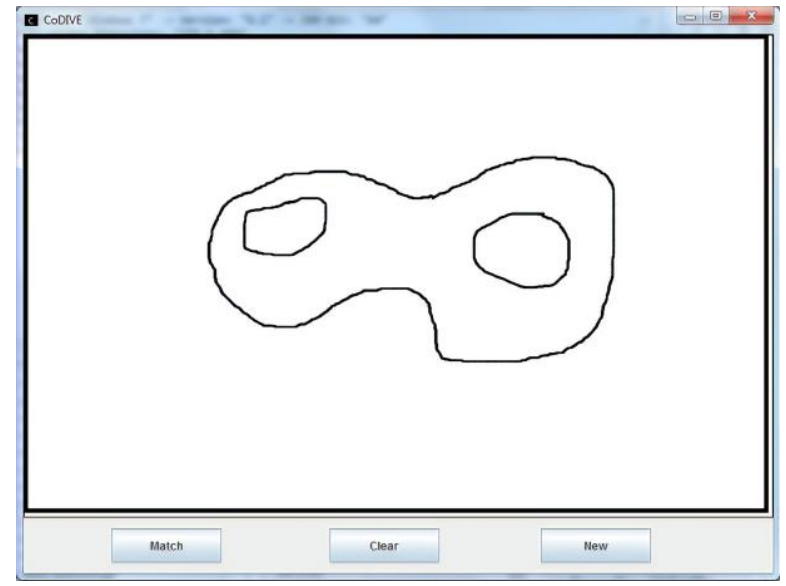

(b)

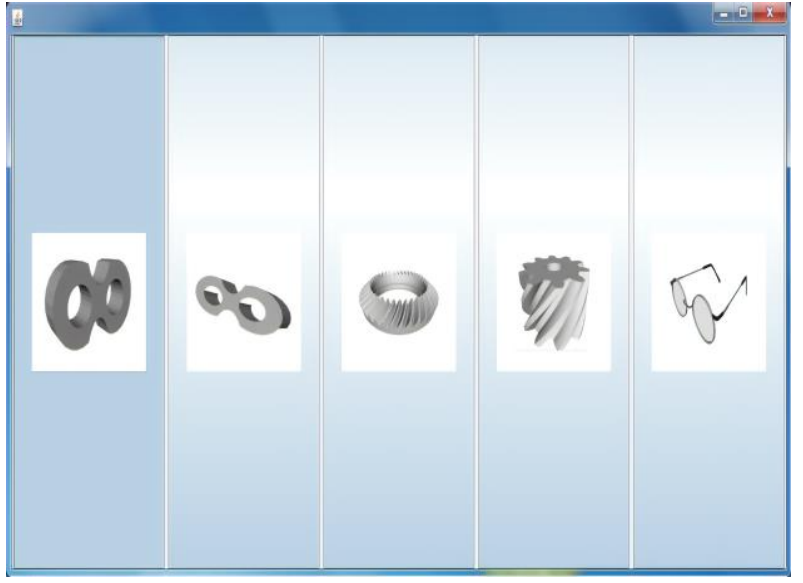

$-2-x$

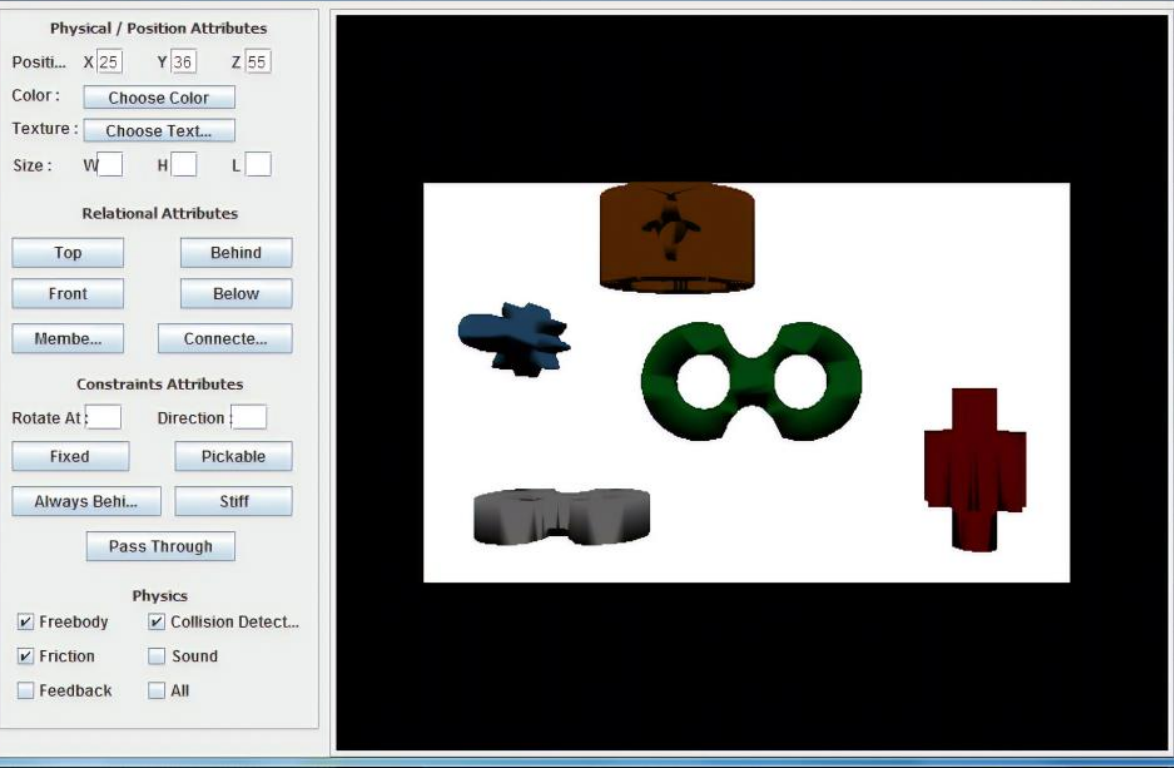

(c) 
Figure 2. (a) The sketching block, (b) Selection of matched objects, (c) VR builder block

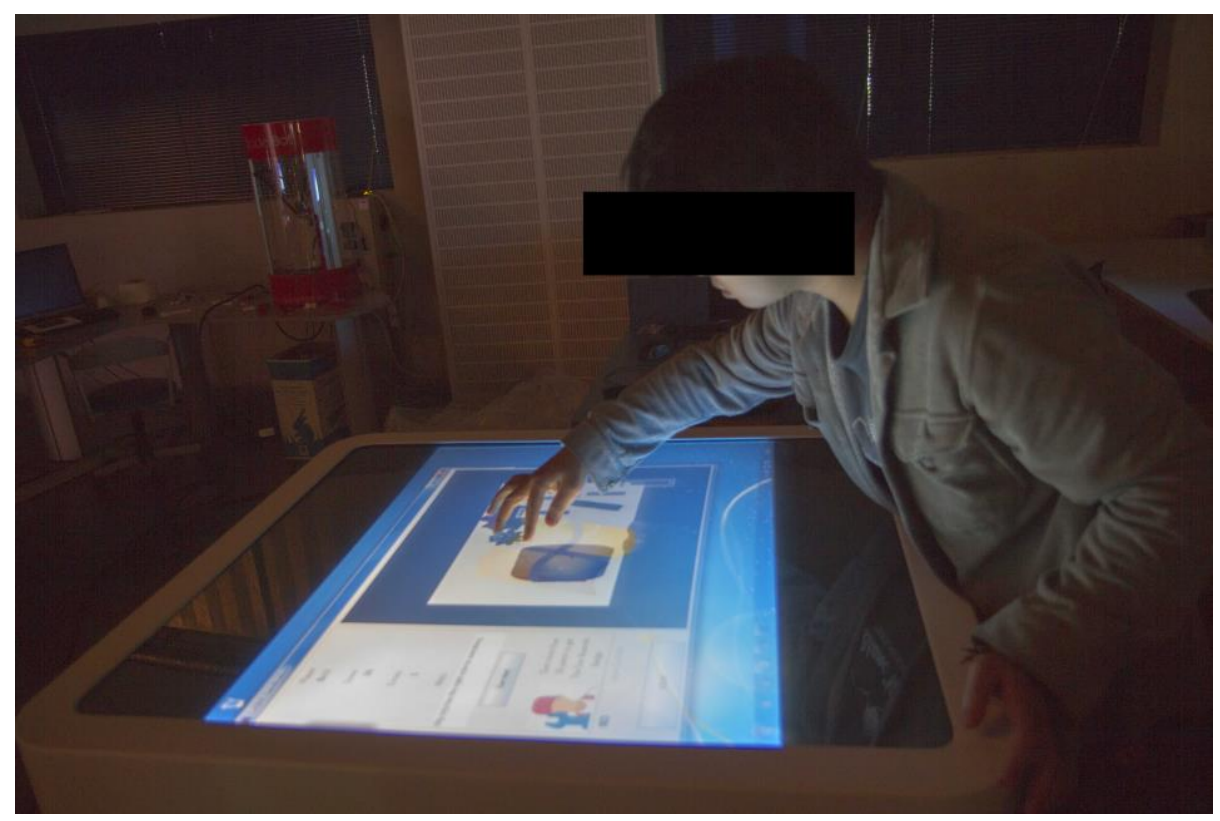

Figure 3. A user engaged in the assembly game on the multi-touch table 


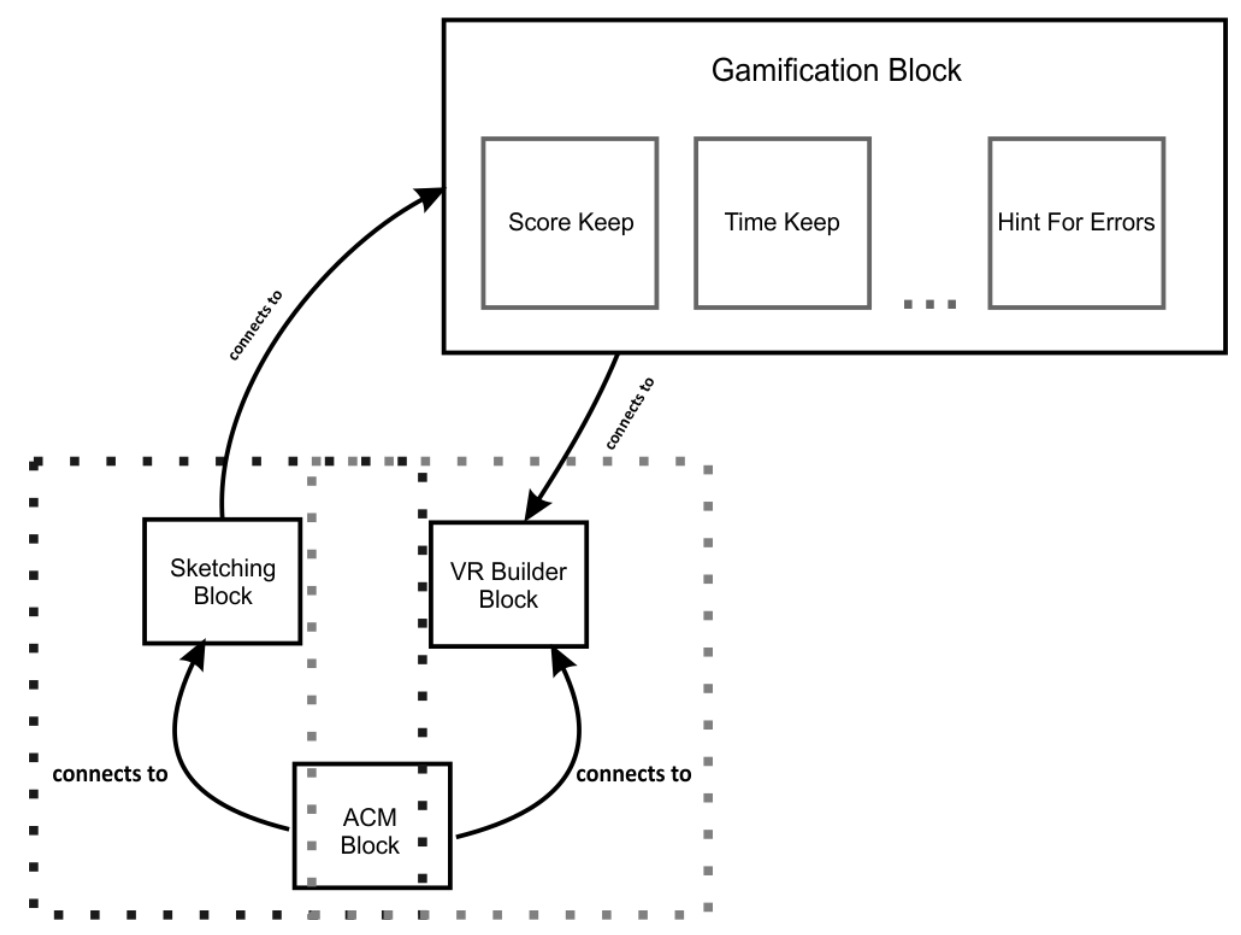

Figure 4. The integration of the gamification concept in Co-DIVE system

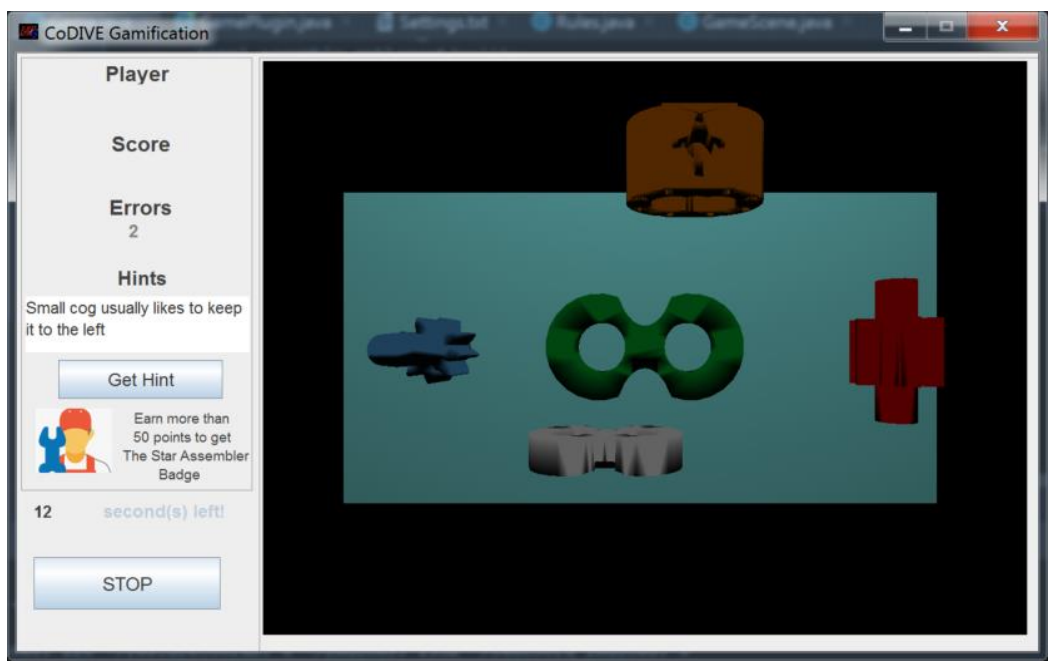

Figure 5. Gamification of the virtual assembly environment 
(a)

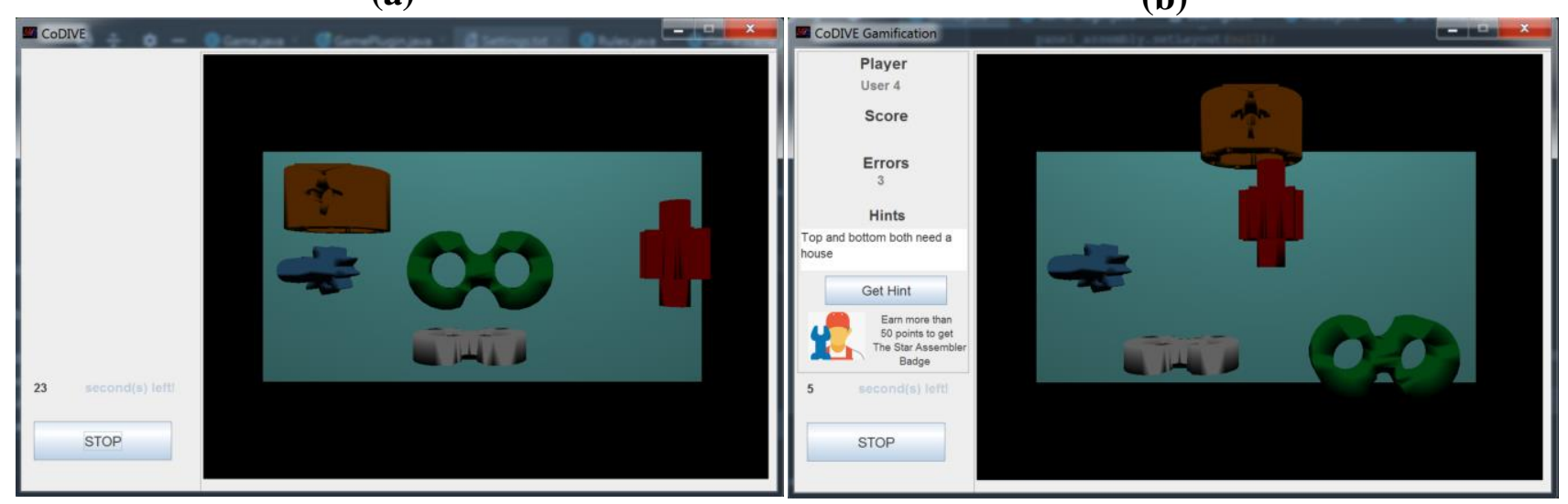

Figure 6. (a) Non-Gamified virtual assembly; (b) Gamified virtual assembly (b)
Number of errors during assembly task

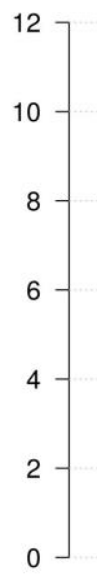

$\left.\begin{array}{l}12 \\ 0- \\ 8 \\ 6 \\ 4 \\ 2 \\ 2 \\ 0\end{array}\right]$

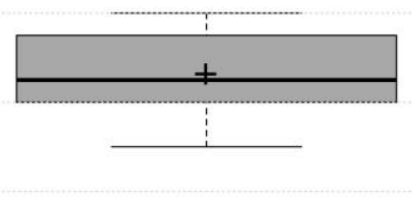

Gamified 


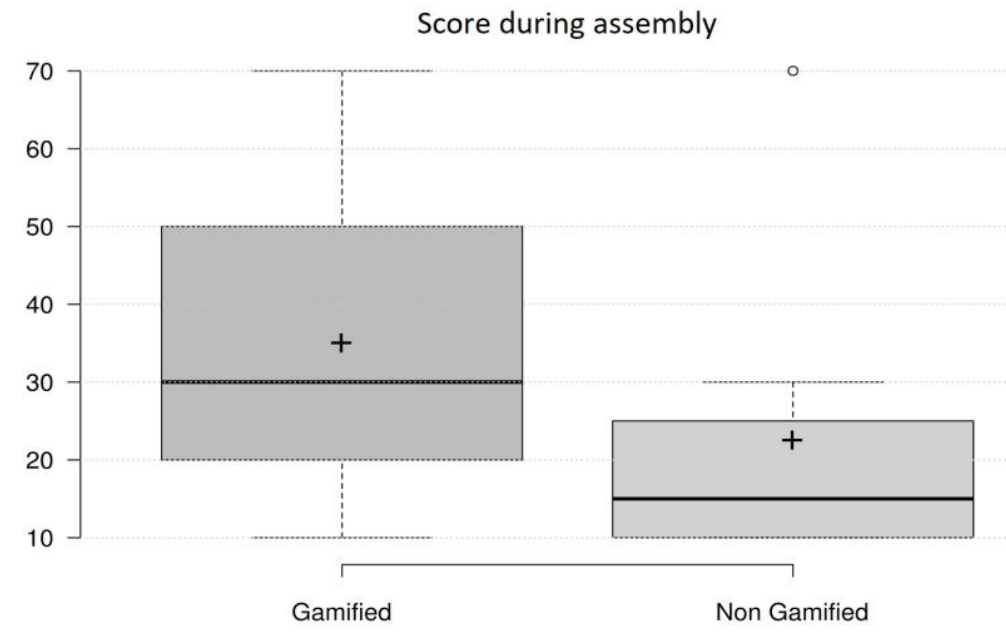

Figure 8. Mean level for scores in the two experimental conditions

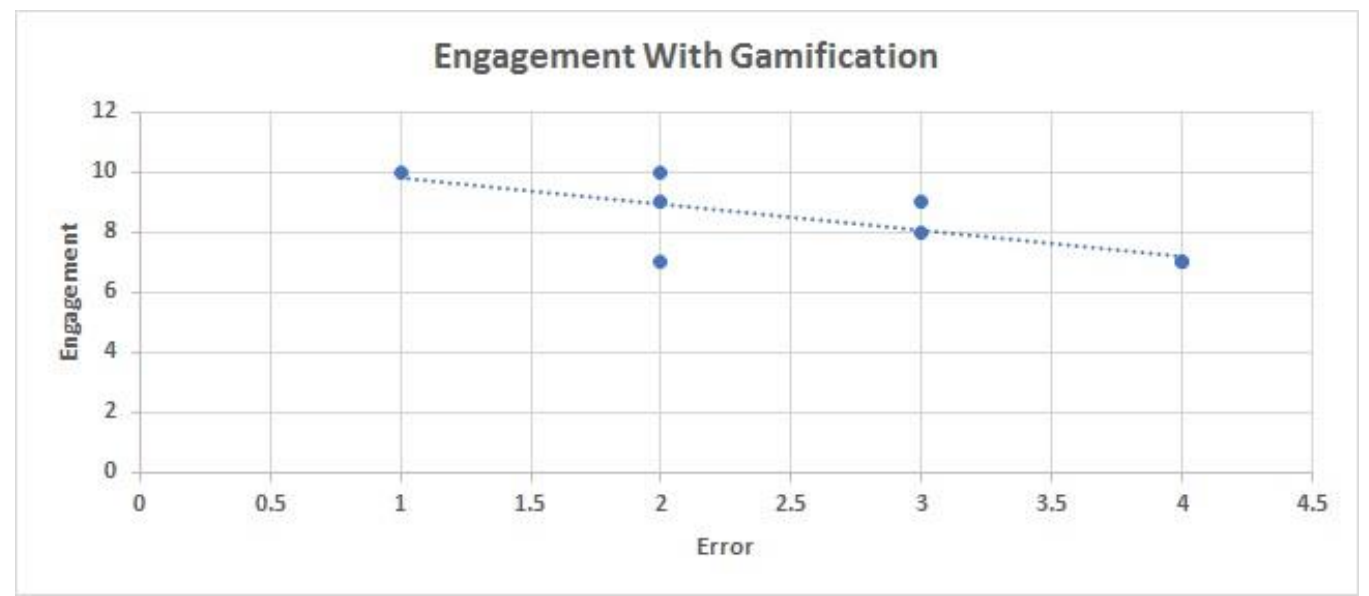

Figure 9. The negative correlation between error and engagement in the gamified condition 


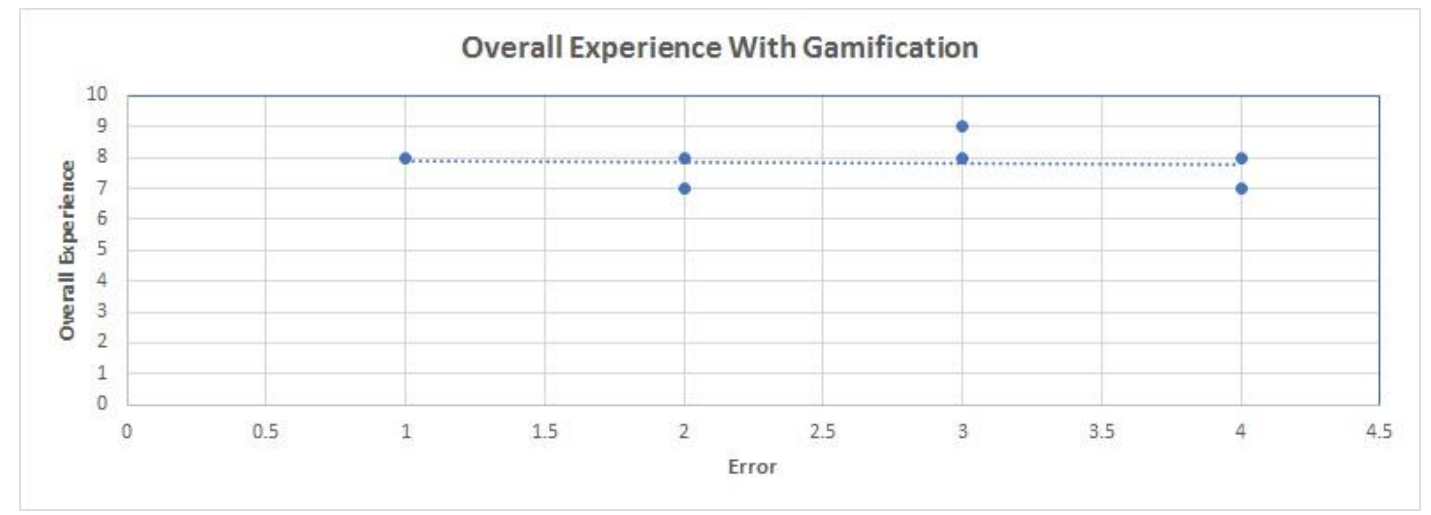

Figure 10. The negative correlation between error and overall experience in the gamified condition

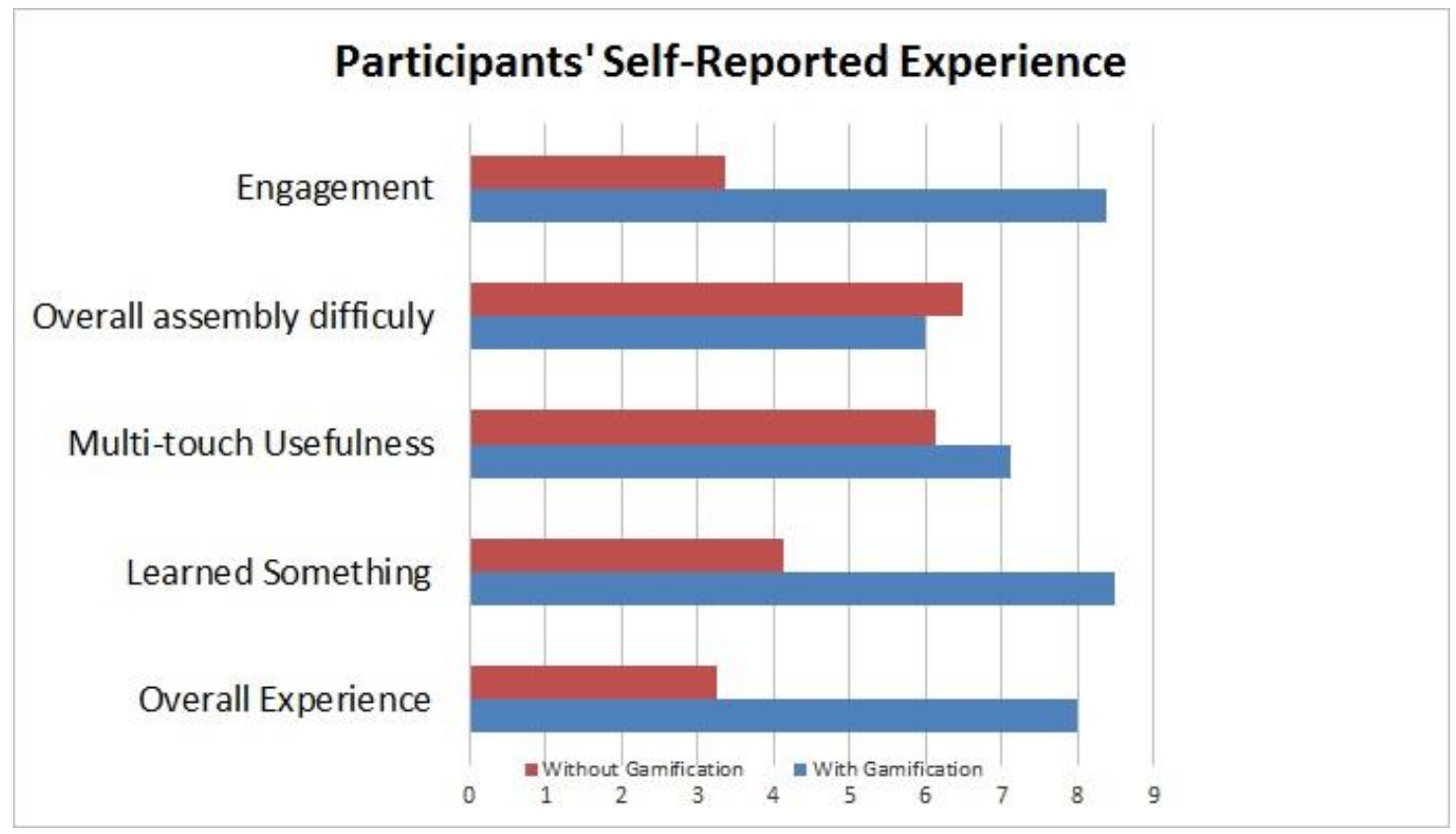

Figure 11. Feedback from users 


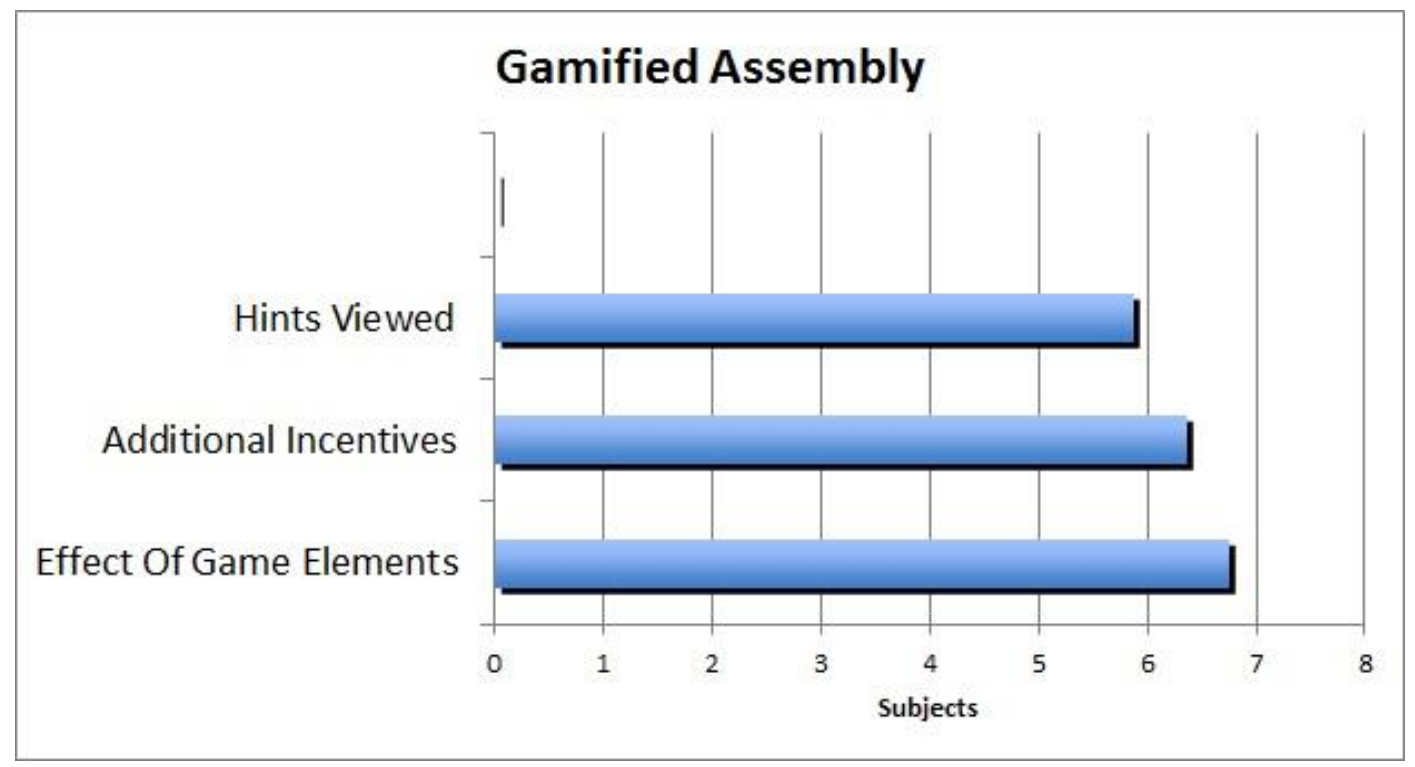

Figure 12. Users' scoring of the assembly game 